\title{
Chronic Diarrhea as the Presenting Complaint of Systemic Lupus Erythematosus in a Man
}

\author{
Ashwin Rajendiran ${ }^{1}$, Stalin Viswanathan ${ }^{2}$, Bhavith Remalayam ${ }^{1}$, \\ Vivekanandan Muthu ${ }^{2}$ and Thomas Alexander ${ }^{3}$
}

\begin{abstract}
Systemic lupus can involve any part of the gastrointestinal (GI) tract. Diarrhea generally results from complications arising from infection, drugs or pancreatitis. We herein report the case of a 40-year-old hypertensive man with a psychotic disorder in whom the evaluation of chronic diarrhea revealed a diagnosis of systemic lupus erythematosus (SLE), diffuse proliferative glomerulonephritis and protein-losing enteropathy that required treatment with both steroids and mycophenolate mofetil. Over the following year, the patient developed atrial fibrillation, miliary tuberculosis and generalized clonic tonic seizures. He is currently under regular follow-up care and receives antiepileptics, antihypertensives, diltiazem, amiodarone and warfarin.
\end{abstract}

Key words: male SLE, gastrointestinal manifestations, diarrhea

(Intern Med 51: 3131-3134, 2012)

(DOI: 10.2169/internalmedicine.51.8518)

\section{Introduction}

The clinical presentation of systemic lupus erythematosus (SLE) in males varies among different populations. The gastrointestinal manifestations of SLE can involve any part of the gastrointestinal (GI) tract, with incidences ranging from $1.3 \%$ to $27.5 \%$ (1-3). Diarrhea occurring in patients with SLE generally results from parasitic or bacterial intestinal infections and can be caused by chronic pancreatitis or drugs such as azathioprine $(4,5)$. Rarer complications of SLE, including protein-losing enteropathy, lupus enteritis (mesenteric vasculitis), intestinal pseudo-obstruction and SLE-associated disorders such as eosinophilic enteritis and celiac disease, can also contribute to diarrhea (6). Diarrhea has been reported to be the initial complaint of SLE in mostly female patients. We herein report the case of a 40year-old man with chronic diarrhea as the presenting complaint of SLE.

\section{Case Report}

The patient presented to the referring hospital due to non- bloody diarrhea lasting for three months with a low grade fever, vomiting and weight loss. Following 10 days of cefotaxime and metronidazole therapy, his symptoms improved. He was referred to our hospital two weeks later after his symptoms recurred. Six years previously, he had been noted to have hypertension and was prescribed metoprolol. Eight months previously, the patient had been diagnosed with paranoid schizophrenia and underwent one session of electroconvulsive therapy. His laboratory results were as follows: hemoglobin: $10.6 \mathrm{~g}$, platelets: $185 \times 10^{9} / \mathrm{L}$, ESR: $89 \mathrm{~mm} / \mathrm{hr}$, urea: $7 \mathrm{mmol} / \mathrm{L}$ and creatinine: $90 \mu \mathrm{mol} / \mathrm{L}$. Since then, he had been taking nitrazepam, oxcarbamazepine, risperidone and metoprolol regularly. He smoked -10 cigarettes/day and consumed $-120 \mathrm{~g}$ of alcohol per week. On admission, he had severe pallor, crusted lips, retromolar mucosal erosions, bilateral pitting pedal edema, bilateral wheezes with infraaxillary and infra-scapular crackles and intention tremors.

The patient's test results are listed in Table. His liver function and electrolyte results were normal. The 24-hour urinary protein level was $770 \mathrm{mg}$. Bacterial and fungal cultures of blood, urine and stools were sterile. Other test results showed fatty liver (ultrasonography), esophagitis and mild duodenal nodularity (esophagogastroduodenoscopy), an

${ }^{1}$ Department of Internal Medicine, Pondicherry Institute of Medical Sciences, India, ${ }^{2}$ Department of General Medicine, Indira Gandhi Medical College, India and ${ }^{3}$ Department of Gastroenterology, Pondicherry Institute of Medical Sciences, India

Received for publication July 4, 2012; Accepted for publication August 15, 2012

Correspondence to Dr. Stalin Viswanathan, stalinviswanathan@ymail.com 
Table. Laboratory Results for the Patient after Admission

\begin{tabular}{lrrrrr}
\hline Investigation & Normal & D1 & D 10 & \multicolumn{1}{c}{ D 15 } & Discharge \\
\hline Hemoglobin $(\mathrm{g} \%)$ & $12-14$ & 5.5 & 7.9 & 7.1 & 9.3 \\
WBC count $\times 10^{9} / \mathrm{L}$ & $4-10$ & 5.1 & 8.2 & 2.7 & 11.7 \\
Platelets $\times 10^{9} / \mathrm{L}$ & $150-450$ & 150 & 140 & 150 & 142 \\
Urea $(\mu \mathrm{mol} / \mathrm{L})$ & $2.5-7$ & 24.64 & 29.92 & 24.29 & 9.85 \\
Creatinine $(\mu \mathrm{mol} / \mathrm{L})$ & $44-80$ & 308 & 325.6 & 237 & 90 \\
Albumin $(\mathrm{g} / \mathrm{L})$ & $40-50$ & 20 & 26 & & 30 \\
ESR $(\mathrm{mm} / \mathrm{hr})$ & $10-20$ & 32 & 30 & & \\
Urine $\mathrm{RBCs} / \mathrm{hpf}$ & $10-15$ & Anti-ds DNA & $>300$ & $<15$ \\
Urine Pus cells/hpf & $10-12$ & ANA & & 5.2 & $<1.0$ \\
Stool ova/cyst & Negative & C3 & & $0.76 \mathrm{~g} / \mathrm{dL}$ & $0.83-1.77$ \\
Stool occult blood & Positive & C4 & & $0.10 \mathrm{~g} / \mathrm{dL}$ & $0.16-0.47$ \\
HIV & Negative & APL & Negative & \\
HBsAg & Negative & ACL & Negative & \\
AntiHCV & Negative & Leptospira IgM & Negative & \\
\hline
\end{tabular}

WBC: white blood cells, ESR: erythrocyte sedimentation rate, HIV: human immunodeficiency virus, HBsAg: hepatitis B surface antigen, ANA: antinuclear antibody, anti-dsDNA: double stranded DNA, C3: complement 3, C4: complement 4, APL: antiphospholipid antibody, ACL: anticardiolipin antibody

anal fissure (colonoscopy) and non-contributory barium-meal follow-through (Figure). Biopsies of the ileum and colon showed lamina propria edema, lymphatic dilatation and chronic inflammatory infiltrates. Lower limb venous Doppler, electrocardiography, echocardiography and computed tomography (CT) of the abdomen were normal. Two packed-RBC transfusions and salt-free albumin were administered after admission. The treatment regimen included risperidone, trihexyphenydyl, liquid paraffin [lip crusts], mupirocin ointment, loperamide and oral rehydration solutions. A renal biopsy revealed diffuse proliferative glomerulonephritis. Following the diagnosis of SLE, treatment with $60 \mathrm{mg}$ of oral prednisolone was initiated after three days of pulse methylprednisolone under imipenem cover. Treatment with mycophenolate mofetil at a dose of $1 \mathrm{~g}$ BID was added after two weeks to treat persistent symptoms with significant relief. Two months following discharge, the patient was readmitted for atrial fibrillation and treated with enoxaparin, diltiazem and amiodarone. He received follow-up care on an outpatient basis and continued to take prednisolone at a dose of $30 \mathrm{mg}$ and mycophenolate mofetil at a dose of $500 \mathrm{mg}$ BID.

One year later, the patient presented with loss of appetite, fatigue and fever lasting 20 days with Medical Research Council (MRC) grade IV dyspnea. With a miliary pattern observed on chest radiography and the patient's refusal to undergo high resolution chest CT, empirical antituberculous treatment was initiated and the symptoms began to resolve. The patient's renal function and diarrhea remained stable. Three months later, he was readmitted after experiencing a generalized tonic clonic seizure that was treated with diazepam and phenytoin. Brain contrast CT and MRI did not reveal any abnormalities. Currently, the patient is taking diltiazem, risperidone, folic acid, amiodarone, telmisartan, carbamazepine and warfarin.

\section{Discussion}

Studies of SLE have shown that men comprise $4-22 \%$ of cases (4). The male/female ratio was reported to be 11.4:1 in a Chinese study (7). Western studies have shown either no differences or conflicting organ involvement between the sexes, except in cases of renal disease, which is more common in men (7). Pande et al., in a study of 39 male SLE patients from India, noted that the prevalence of arthritis, mucosal lesions and alopecia was high, whereas that of hematological abnormalities, psychosis, diffuse proliferative nephritis and gastrointestinal involvement were low (4). The latter three complications comprised the initial manifestations in our patient. In a study of the largest cohort of male SLE patients, no GI manifestations or psychosis were observed at the onset of the disease (8). Mucosal symptoms, including ulcers, anorexia and abdominal pain, are common, while diarrhea, fecal incontinence, perforation and hemorrhage are rare $(1,2,9)$. Other very uncommon abdominal manifestations in SLE patients include protein-losing enteropathy (PLE), lupus enteritis, eosinophilic enteritis and intestinal pseudo-obstruction (IPO) (3). Drugs and infection contribute most to the development of GI disease, in contrast to renal involvement, which is typically caused by disease activity (6). Among Indian males with SLE, GI manifestations occur at an incidence of $15 \%$ (4). The Asian population has a higher prevalence (18\%) of GI manifestations compared to populations on other continents (2). Hepatosplenomegaly is the only GI manifestation that is more common in men (7) and that has been observed in the Israeli population. GI complications develop more commonly in SLE patients with hematological or renal manifestations or hypocomplementemia and in those who are positive for Raynaud's phenomenon (9).

Diarrhea as a manifestation of SLE was observed in 37\% to $45 \%$ of cohorts in three large studies (2). Forty-six cases (six men) of SLE with PLE have been reported. PLE was reported to be the initial presentation in 30 of the 46 patients: 17 had diarrhea and 24 had either ascites or pleural or pericardial effusion (2). Only one case involving a 24year-old man who presented with PLE and serosal effusion on initial presentation has been reported. That patient also had associated primary sclerosing cholangitis. The exact pathophysiologic mechanisms of PLE have not been elucidated; however, causes of PLE have been attributed to vasculitis, complement deposition, chronic infection and inflammatory bowel diseases that disrupt lymphatic vessels. Patients with PLE present with severe pitting edema, diarrhea, hypoalbuminemia and hypocomplementemia. Patients with PLE have increased stool antitrypsin levels and high stool losses of radiolabeled albumin (10). Tc-99 albumin scintigraphy, the gold standard for diagnosing PLE was not performed in this case due to unavailability. Since the results of colonoscopy, ultrasonogram and abdominal CT were normal in this patient, diagnoses of IPO and lupus enteritis (mesen- 

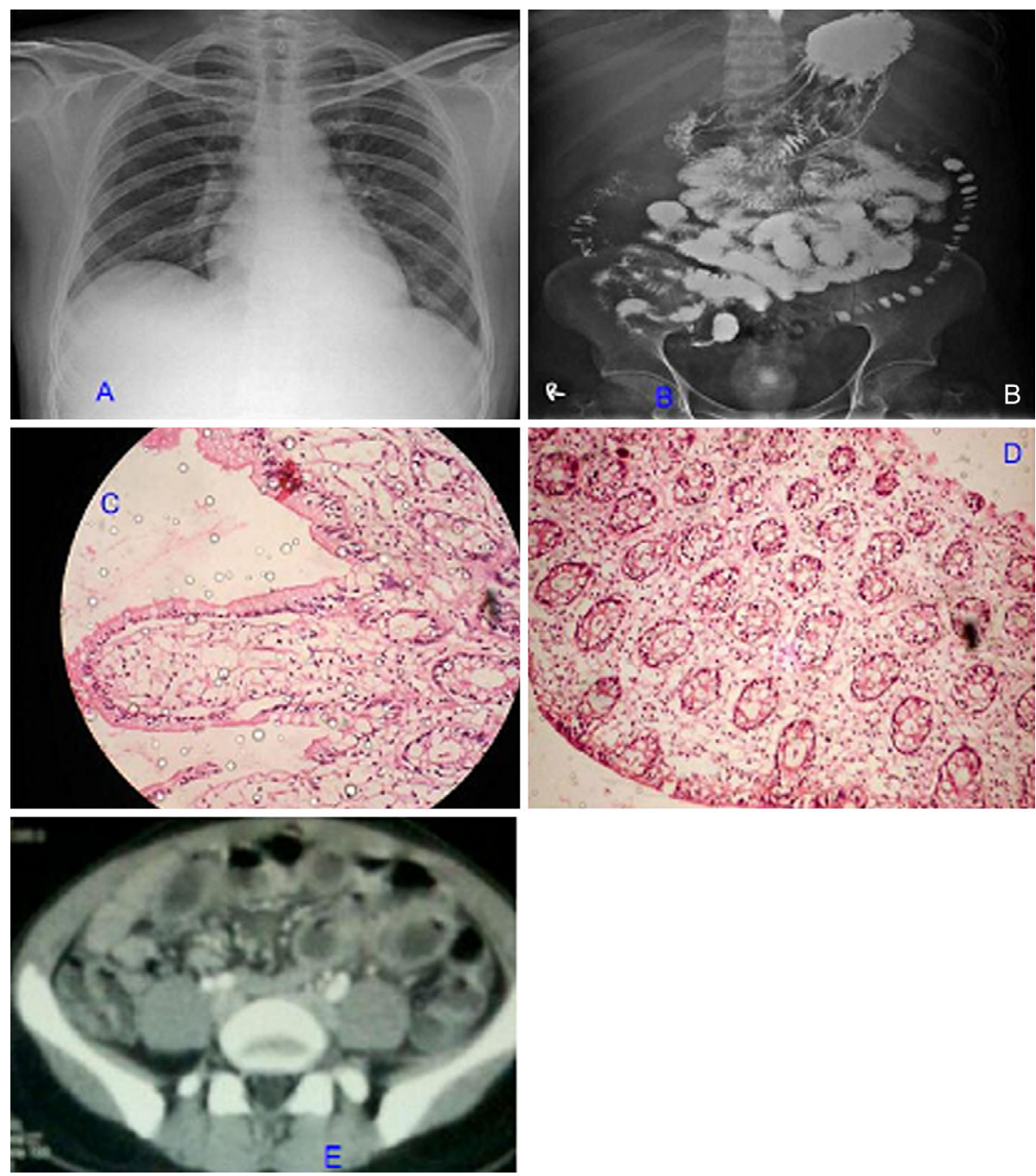

Figure. A: CXR performed at admission showing straightened of heart border. B: Barium-meal follow-through showing a normal mucosal appearance of jejunum and ileum. C: An ileal biopsy $(40 \times)$ showing mild edema of lamina propria with lymphoid aggregates and dilated lymphatic channels. D: A colonic biopsy(200x) showing an edematous lamina propria with mild chronic inflammation without crypt distortion. E: Abdominal CT showing normal fluid-filled bowel loops without wall thickening or "double halo" sign.

teric vasculitis) can be ruled out. Other causes of diarrhea, including pancreatitis, eosinophilic enteritis, pneumatosis cystoides intestinalis and celiac disease were unlikely given that radiology and intestinal biopsies were noncontributory. The results of the intestinal biopsies performed in our patient were suggestive of lymphocytic colitis, NSAID use or autoimmune disease (11). Infection (bacterial, viral and parasitic) and inflammatory bowel disease are common causes of chronic diarrhea in India, and awareness of this complaint as an initial manifestation of SLE is low. Lupus nephritis- and malnutrition (poor intake)-related hypoalbuminemia could also have contributed to the pedal edema observed in our patient. Sixty percent of patients with PLE generally improve with steroid therapy (6). Others require additional immunosuppressants such as azathioprine and cyclophosphamide. Our patient received mycophenolate mofetil to treat severe renal disease, which also resulted in the remission of diarrhea.
In conclusion, GI manifestations in patients with SLE are generally mild and easily overlooked. Features of PLE such as pedal edema, ascites, pleural effusion, dyslipidemia and hypocomplementemia can mimic lupus nephritis if not associated with diarrhea. Chronic diarrhea due to PLE as the presenting manifestation of SLE is very rare in men, and a diagnosis of SLE should be kept in mind when other systemic manifestations are absent.

The authors state that they have no Conflict of Interest (COI).

\section{References}

1. Akitake R, Nakase H, Ueno S, Miyamoto S, Iehara N, Chiba T. Involvement of lupus enteritis in a patient with lupus cystitis and nephritis. Digestion 80: 160-164, 2009.

2. Chng HH, Tan BE, Teh CL, Lian TY. Major gastrointestinal manifestations in lupus patients in Asia: lupus enteritis, intestinal 
pseudo-obstruction, and protein-losing gastroenteropathy. Lupus 19: 1404-1413, 2010.

3. Sunkureddi PR, Luu N, Xiao SY, Tang WW, Baethge BA. Eosinophilic enteritis with systemic lupus erythematosus. South Med J 98: 1049-1052, 2005.

4. Pande I, Malaviya AN, Sekharan NG, Kailash S, Uppal SS, Kumar A. SLE in Indian men: analysis of the clinical and laboratory features with a review of the literature. Lupus 3: 181-186, 1994.

5. Santiago M. Diarrhoea secondary to azathioprine in two patients with SLE. Lupus 8: 565, 1999.

6. Tian XP, Zhang X. Gastrointestinal involvement in systemic lupus erythematosus: insight into pathogenesis, diagnosis and treatment. World J Gastroenterol 16: 2971-2977, 2010.

7. Mok CC, Lau CS, Chan TM, Wong RW. Clinical characteristics and outcome of southern Chinese males with systemic lupus erythematosus. Lupus 8: 188-196, 1999.

8. Garcia MA, Marcos JC, Marcos AI, et al. Male systemic lupus erythematosus in a Latin-American inception cohort of 1214 patients. Lupus 14: 938-946, 2005.

9. Xu D, Yang H, Lai CC, et al. Clinical analysis of systemic lupus erythematosus with gastrointestinal manifestations. Lupus 19: 866869, 2010.

10. Ebert EC, Hagspiel KD. Gastrointestinal and hepatic manifestations of systemic lupus erythematosus. J Clin Gastroenterol 45: 436-441, 2011.

11. Carpenter HA, Talley NJ. The Importance of clinicopathological correlation in the diagnosis of inflammatory conditions of the colon: histological patterns with clinical implications. Am J Gastroenterol 95: 878-896, 2000.

(C) 2012 The Japanese Society of Internal Medicine http://www.naika.or.jp/imonline/index.html 